\title{
SAFIUS - A secure and accountable filesystem over untrusted storage
}

\author{
V Sriram, Ganesh M. Narayan, K Gopinath \\ Computer Science and Automation \\ Indian Institute of Science, Bangalore \\ $\{$ v_sriram,nganesh, gopi\}@csa.iisc.ernet.in
}

\begin{abstract}
We describe SAFIUS, a secure accountable file system that resides over an untrusted storage. SAFIUS provides strong security guarantees like confidentiality, integrity, prevention from rollback attacks, and accountability. SAFIUS also enables read/write sharing of data and provides the standard UNIX-like interface for applications. To achieve accountability with good performance, it uses asynchronous signatures; to reduce the space required for storing these signatures, a novel signature pruning mechanism is used. SAFIUS has been implemented on a GNU/Linux based system modifying OpenGFS. Preliminary performance studies show that SAFIUS has a tolerable overhead for providing secure storage: while it has an overhead of about $50 \%$ of OpenGFS in data intensive workloads (due to the overhead of performing encryption/decryption in software), it is comparable (or better in some cases) to OpenGFS in metadata intensive workloads.
\end{abstract}

\section{INTRODUCTION}

With storage requirements growing at around $40 \% \mathrm{ev}-$ ery year, deploying and managing enterprise storage is becoming increasingly problematic. The need for ubiquitous storage accessibility also requires a re-look at traditional storage architectures. Organizations respond to such needs by centralizing the storage management: either inside the organization, or by outsourcing the storage. Though both the options are together feasible and can coexist, they both pose serious security hazards: the user can no longer afford to implicitly trust the storage or the storage provider/personnel with critical data.

Most systems respond to such a threat by protecting data cryptographically ensuring confidentiality and integrity. However, conventional security measures like confidentiality and update integrity alone are not sufficient in managing long lived storage: the storage usage needs to be accounted, both in quality and quantity; also the inappropriate accesses, as specified by the user, should be disallowed and individual accesses should ensure non-repudiation. In order for such storage to be useful, the storage accesses should also provide freshness guarantees for updates.

In this work we show that it is possible to architect such a secure and accountable file system over an untrusted storage which is administrated in situ or outsourced. We call this architect SAFIUS: SAFIUS is designed to leverage trust onto an easily manageable entities, providing secure access to data residing on untrusted storage. The critical aspect of SAFIUS that differentiates it from rest of the solutions is that storage clients themselves are independently managed and need not mutually trust each other.

\section{A. Data is mine, control is not!}

In many enterprise setups, users of data are different from the ones who control the data: data is managed by storage administrators, who are neither producers nor consumers of the data. This requires the users to trust storage administrators without an option. Increased storage requirements could result in an increase in the number of storage administrators and users would be forced to trust a larger number of administrators for their data. A survey ${ }^{1}$, by Storagetek, revealed that storage administration was a major cause of difficulty in storage management as data storage requirements increased. Although outsourcing of storage requirements is currently small, with continued explosion in the data storage requirements and sophistication of technologies needed to make the storage efficient and secure, enterprises may soon outsource their storage (management) for cost and efficiency reasons. Storage service providers (SSPs) provide storage and its management as a service. Using outsourced storage or storage services would mean that entities outside an enterprise have access to (and in fact control) enterprise's data.

\section{B. Need to treat storage as an untrusted entity}

Hence, there is a strong need to treat storage as an untrusted entity. Systems like PFS[7], Ivy[5], SUNDR[3], Plutus[4] and TDB[9] provide a secure filesystem over an untrusted storage. Such a secure filesystem needs to provide integrity and confidentiality guarantees. But, that alone is not sufficient as the server can still disseminate

\footnotetext{
${ }^{1}$ http://www.storagetek.com.au/company/press/survey.html
} 
old, but valid data to the users in place of the most recent data (rollback attack [4]). Further, the server, if malicious, cannot be trusted to enforce any protection mechanisms (access control) to prevent one user from dabbling with another user's data which he is not authorized to access. Hence a malicious user in collusion with the server can mount a number of attacks on the system unless prevented.

All systems mentioned above protect the clients from the servers. But, we argue that we also need to protect the server from malicious clients. If we do not do this, we may end up in a situation where the untrusted storage server gets penalized even when it is not malicious. If the system allows arbitrary clients to access the storage, then it would be difficult to control each of these clients to obey the protocol. The clients themselves could be compromised or the users who use the clients could be malicious. Either way the untrusted storage server could be wrongly penalized. To our knowledge, most systems implicitly trust the clients and may not be useful in certain situations.

\section{SAFIUS - Secure Accountable filesystem over un- trusted storage}

We propose SAFIUS, an architecture that provides accountability guarantees apart from providing secure access to data residing on untrusted storage. By leveraging on an easily manageable trusted entity in the system, we provide secure access to a scalable amount of data (that resides on an untrusted storage) for a number of independently managed clients. The trusted entity is needed only for maintaining some global state to be shared by many clients; the bulk data path does not involve the trusted entity. SAFIUS guarantees that a party that violates the security protocol can always be identified precisely, preventing entities which obey the protocol from getting penalized. The party can be one of the clients which exports filesystem interface to users or the untrusted storage.

The following are the high level features of SAFIUS

- It provides confidentiality, integrity and freshness guarantees on the data stored.

- It can identify the entities that violate the protocol.

- It provides sharing of data for reading and writing among users.

- Clients can recover independently from failures without affecting global filesystem consistency.

- It provides close to UNIX like semantics.

The architecture is implemented in GNU/Linux. Our studies show that SAFIUS has a tolerable overhead for providing secure storage: while it has an overhead of about $50 \%$ of OpenGFS for data intensive workloads, it is comparable (or better in some cases) to OpenGFS in metadata intensive workloads.

\section{DESIGN}

SAFIUS provides secure access to data stored on an untrusted storage with perfect accountability guarantees by maintaining some global state in a trusted entity. In the SAFIUS system, there are fileservers ${ }^{2}$ that provide filesystem access to clients, with the back-end storage residing on untrusted storage, henceforth referred to as storage server. The fileservers can reach the storage servers directly. The system also has lock servers, known as l-hash server (for lock-hash server), a trusted entity that provides locking service and also holds and serves some critical metadata.

\section{A. Security requirements}

Since the filesystem is built over an untrusted data store, it is mandatory to have confidentiality, integrity and freshness guarantees for the data stored. These guarantees prevent the exposure or update of data by the storage server either by unauthorized users or by collusion between unauthorized users and the storage server. Wherever there is mutual distrust between entities, protocols employed by the system should be able to identify the misbehaving entity (entity which violates the protocol) precisely. This feature is referred to as accountability.

\section{B. Sharing and Scalability}

The system should enable easy and seamless sharing of data between users in a safe way. Users should be able to modify sharing semantics of a file on their own, without the involvement of a trusted entity. The system should also be scalable to a reasonably large number of users.

\section{Failures and recoverability}

The system should continue to function, tolerating failures of the fileservers and it should be able to recover from failures of 1-hash servers or storage servers. Fileservers and storage servers can fail in a byzantine manner as they are not trusted and hence can be malicious. The fileservers should recover independently from failures.

\section{Threat Model}

SAFIUS is based on a relaxed threat model:

- Users need not trust all the fileservers uniformly. They need to trust only those fileservers through which they access the filesystem. Even this trust is temporal and can be revoked. It is quite impractical to build a system without the user to fileserver trust ${ }^{3}$.

\footnotetext{
${ }^{2}$ They are termed fileservers as these machines can potentially serve as NFS servers with a looser consistency semantics to end clients.

${ }^{3}$ The applications which access the data would not have any assurance on the data read or written as it passes through an untrusted operating system
} 


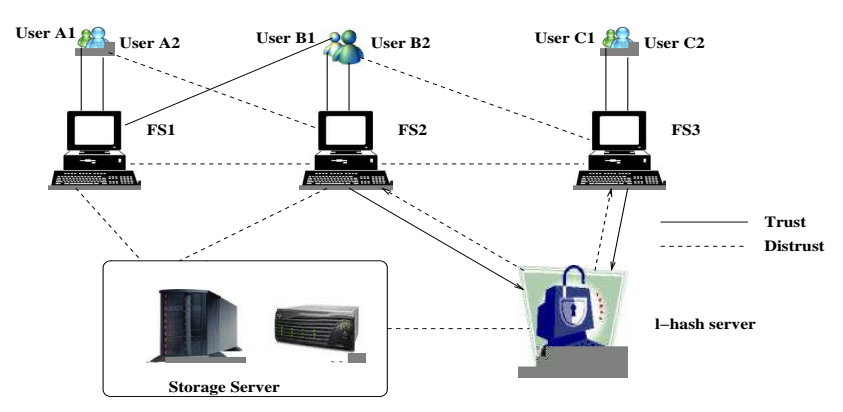

Fig. 1. Threat Model

- No entity trusts the storage server and vice versa. The storage server is not even trusted for correctly storing of data.

- The users and hence the fileservers need not trust each other and we assume that they do not. This assumption is important for ease of management of the fileservers. The fileservers can be independently managed and the users have the choice and responsibility to choose which fileservers to trust.

- The 1-hash servers are trusted by the fileservers, but not vice versa.

Figure 1 illustrates an instance of this threat model. Users $A 1$ and $A 2$ trust the fileserver $F S 1$, users $B 1$ and $B 2$ trust the fileserver $F S 2$ and users $C 1$ and $C 2$ trust the fileserver $F S 3$. User $B 1$ apart from trusting $F S 2$ also trusts $F S 1$.

If we consider trust domains ${ }^{4}$ to be made of entities that trust each other either directly or transitively then SAFIUS guarantees protection across trust domains. The trust relationship could be limited in some cases (sharing of few files) or it could be complete (user trusting a fileserver). This threat model provides complete freedom of administering the fileservers independently and hence eases the manageability.

\section{ARCHITECTURE}

The block diagram of the SAFIUS architecture is shown in figure 2. Every fileserver in the system has a filesystem module that provides the VFS interface to the applications, a volume manager through which the filesystem talks to the storage server and a lock client module that interacts with the l-hash server for obtaining, releasing, upgrading, or downgrading of locks. The 1-hash server, apart from serving lock requests, also distributes the hash of inodes. The 1-hash server also has the filesystem module, volume manager module and a specialized version of lock client module and can be used like any other fileserver in the

\footnotetext{
${ }^{4}$ If we treat the entities in the system as nodes of a graph and an edge between $i$ and $j$, if $i$ trusts $j$, then each connected component of the graph forms a trust domain
}

system. The lock client modules do not interact directly among each other, as they do not have mutual trust. The lock clients interact transitively through the 1-hash server which validates the requests. The fileservers can fetch the hash of inode from the trusted 1-hash server and hence fetch any file block with integrity guarantees. In figure 2 , the thick lines represent bulk data path and the thin lines the metadata path. This model honours the trust assumptions stated earlier and can scale well because each fileserver talks to the block storage server directly.

\section{A. Block addresses, filegroups and inode-table in SAFIUS}

The blocks are addressed by their content hashes similar to systems like SFS-RO [1]. It gives a write-once property and blocks cannot be overwritten without changing the pointer to the block. SAFIUS currently uses SHA-1 as the content hash and assumes that SHA- 1 collisions $d$ o not happen. SAFIUS uses the concept of filegroups [4], to reduce the amount of cryptographic keys that need to be maintained. Since "block numbers" are content-hashes, fetching the correct inode block would ensure that the file data is correct. SAFIUS guarantees the integrity of the inode block by storing the hash of the inode block in an inode hash table $i$-tbl. Each tuple of $\mathrm{i}$-tbl is called as idata, and consists of the inode's hash and an incarnation number. $i$ $t b l$ is stored in the untrusted storage server; its integrity is guaranteed by storing the hash of the i-tbl's inode block in a local stable storage in the l-hash server.

\section{B. On-Disk structures: Inode and directory entry}

Inode The inode of a file contains pointers to data blocks either directly or through multiple levels of indirection, apart from other meta information found in standard UNIX filesystems. The block pointers are SHA-1 hashes of the blocks. These apart, it also contains a 4 byte filegroup id, that points to relevant key information to encrypt/decrypt the blocks of this file.

The hash of an inode corresponds to the current version of the file. If a file is updated, then one of its leaf data block changes and hence its intermediate metablocks (as 
it has a pointer to this leaf block) and ultimately the inode block changes (this is similar to what happens in some log structured filesystems, where writes are not done in-place, like wafl [2]). Thus, updating a file can be seen as moving from one version of the file to another, with the version switch happening at a point in time when the file's idata is updated in the i-tbl.

Directory entry Directory entries in SAFIUS are similar to the directory entries in traditional filesystems. They contain a name and the inode number corresponding to the name.

\section{Storage Server}

The granularity at which the storage server serves data is variable sized blocks. The storage server supports three basic operations, namely load, store and free of blocks. A block can be stored multiple times, i.e. clients can issue any number of store requests to the same block and the block has to be freed that many times before the physical block can be reused at the server. To prevent one user from freeing a block belonging to another user, the storage server maintains a per inode number reference count on each of the stored blocks. Each block contains a list of inode numbers and their reference counts. Architectures like SUNDR [3] maintain a per user reference count for the blocks. Having a per user reference count decimates the possibility of seamless sharing which is one of our design goals. For write sharing a file between two users $A$ and $B$ in SUNDR, the users $A$ and $B$ must belong to a group $G$ and the file is write shareable in the group $G$. This group $G$ has to be created and its public key need to be distributed by a trusted entity. This restriction is due to per user reference count on the blocks and a per user table mapping inode numbers to their hashes. Let users $A$ and $B$ write share a file $f$ in SUNDR. If $B$ modifies a block $k$ stored by $A$ earlier to $l$, then $B$ cannot free $k$, as it had not stored it. SAFIUS has a per inode reference count on blocks, and the storage server does the necessary access control to the reference count updates by looking up the filegroup information for sharing information. The trusted l-hash server ratifies the access control enforced by the storage server.

The storage server authenticates the user (through public key mechanisms) who performs the store or free operation. If the uid (of the user) performing the store or free operation is same as the owner of the inode, then the operation is valid. If this is not the case, then the storage server has to verify if the current uid has enough permissions to write to the file. If this check is not enforced, then an arbitrary user can free the blocks belonging to files for which he has no write access. The storage server achieves this by maintaining a cache of inode numbers and their corresponding filegroup ids. This cache is populated and maintained with the help of storage server. This enables seamless write sharing in SAFIUS.

\section{D. l-hash server: $i$-tbl, filegroup tree}

The 1-hash server provides the basic locking service, stores and distributes the idata of inodes to/from the itbl ${ }^{5}$. The l-hash server also maintains a map of inode number to filegroup id information in a fgrp $^{6}$ (filegroup) tree that contains the filegroup data in the leaf blocks of the tree. In addition, there is a persistent 64 bit monotonically increasing fgrp incarnation number, a global count that indicates the number of changes made to file sharing attributes. The root of the filegroup Merkle tree and the fgrp incarnation number are stored locally in the l-hash server. The root of the frrp tree is hashed with the fgrp incarnation number to get fgrp hash.

\section{E. Volume Manager}

The volume manager does the job of translating the read, write, and block free requests from the fileserver to load, store or free operations that can be issued to the storage server. The volume manager exports the standard block interface to the filesystem module, but expects the filesystem module to pass some additional information like hash of an existing block (for reading and freeing) and filegroup id of the block (for encryption or decryption).

\section{F. Encryption and hashing}

The blocks are decrypted and encrypted, as they enter and leave the fileserver machine respectively by the volume manager. On a write request the block is encrypted, hashed and stored. On a read request the blocks are fetched from the storage server, checked for integrity (by comparing the block's hash with that of its pointer) and is decrypted and handed over to the upper layer. The choice of doing the encryption and decryption at the volume manager layer was done to simplify the filesystem implementation and for performance reasons; to delay encryption and avoid repeated decryptions on the same block.

\section{G. Need for non-repudiation}

Since the volume manager and the storage server are mutually distrustful, we need to protect them from the other party's malicious actions:

1. Load Misses: The volume manager requests a block to be a loaded but the storage server replies back saying

\footnotetext{
${ }^{5}$ i-tbl is the persistent table indexed by inode number and contains the i-data corresponding to an inode

${ }^{6}$ It can be realized as a file in SAFIUS
} 


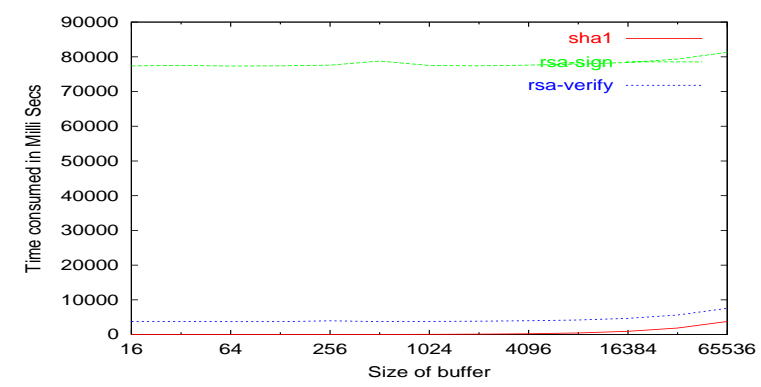

Fig. 3. RSA signing over various block sizes

that the block is not found. It could be that the fileserver is lying (did not store the block at all) or the storage server is lying.

2. Unsolicited stores: A block would be accounted in a particular user's quota, but the user can claim that he never stored the block.

The first case is more serious as there is potential data loss. Load operations do not alter the state of stored data and the fileserver would require the necessary key to decrypt it. However, for obvious reasons, both store and free operations have to be non-repudiating. We achieve this by tagging each of store and free operation with a signature.

Let $D_{u}=\{$ blknum, ino, uid, op, nonce, count $\}$. For a store or free operation, the volume manager sends $\left\{D_{u},\left\{D_{u}\right\}_{K_{u}^{-1}}\right\}$ as the signature. Here blknum refers to the hash of the block that is to be stored or freed, ino refers to the inode number to which the block belongs uid refers to the user id of the user who is performing the operation, nonce is a random number that is unique across sessions and is established with the storage server, count is the count of the current operation in this session and $K_{u}{ }^{-1}$ is the private key of the user. $\left\{D_{u}\right\}_{K_{u}^{-1}}$ is $D_{u}$ signed by the private key of the user. The count is incremented on every store or free operation. The nonce distinguishes two stores or frees to the same block which happens in two different sessions, while count distinguishes two stores or frees to the same block in the same session, hence allowing any number of retransmissions. This signature captures the current state of the operation in the volume manager. The signature is referred to as request signature.

The storage server receives the signature, validates the count, uid and nonce, and verifies the signature. It follows the protocol described earlier to store or free the block. On a successful operation, it prepares and sends a reply signature. Let $D_{s s}=\left\{D_{u}, f g r p h a s h\right\}$. The storage server sends $\left\{D_{s s},\left\{D_{s s}\right\}_{K_{s s}-1}\right\}$ to the volume manager. $D_{u}$ is the same as what the storage server received from the volume manager. $K_{s s}{ }^{-1}$ refers to the private key of the storage server. The volume manager verifies that $D_{u}$ it receives from the storage server is same as the one it had

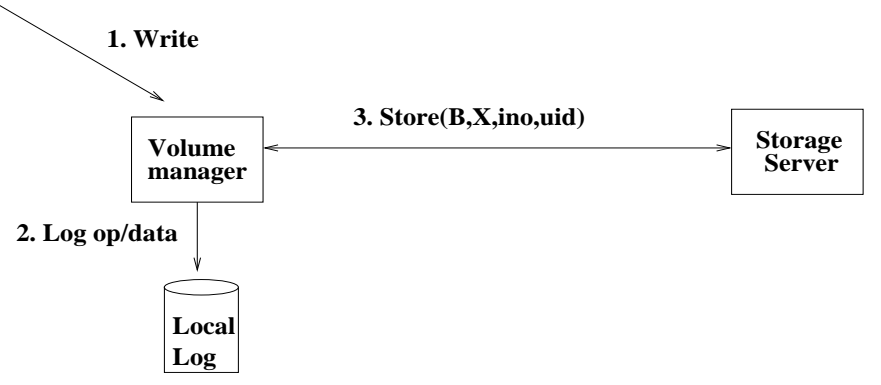

Fig. 4. Sequence of events on a Write

sent and verifies the signature. The signature returned to the volume manager is referred as the grant signature.

The grant signature prevents the storage server from denying the stores made to a block, and in case there were free operations on the block that resulted in the block being removed, the request signature of the free operation would defend the storage server. Unsolicited stores are eliminated as the storage server will not have request signatures for those blocks. Hence assuming that that the RSA signatures are not forgeable, the protocol achieves non-repudiation and hence provides perfect accountability in SAFIUS.

\section{H. Asynchronous signing}

The protocol described above has a huge performance overhead: two signature generations and two verifications in the path of a store or a free operation. Since signature is generated on the hash of a block rather than the block itself, the time taken for actual signature generation, up to a certain block size, masks the time taken for generating the SHA-1-hash of the block. Figure 3 illustrates this. The amount of time taken for signing a 32 byte block and the amount of time taken for signing a 16KB are comparable. However with higher block sizes, the SHA-1 cost shows up and the signature generation cost increases linearly, as can be seen for block sizes bigger than 16KB.

Instead of signing every operation, the protocol signs groups of operations. The store and the free operations do not have signing or verification in their code path and the cost of the signing is amortized among number of store and free operations. Let $B_{u}=$ $\{$ blknum, ino, op, nonce, count $\}$, The fields in this structure are same as that was in $D_{u}$. uid field is not included in $B_{u}$ as we group only operations belonging to a particular user together and it is specified in a header for the signature block. After a threshold number of operations or after a timeout by default, the volume manager packs blocks of $B_{u} \mathrm{~s}^{7}$ in to a block $B D_{u}$. The

\footnotetext{
${ }^{7}$ It contains blocks $B_{u}, B_{u}{ }^{\prime}, B_{u}{ }^{\prime \prime}$
} 
server has signed the root of the tree that it had generated, there cannot be a load miss for a valid block from the storage server side. The refcnt tree in the 1-hash server helps provide accountability. To save some space, the refcnt tree does not store the reference count map for all blocks. It has a table of unique reference count entries (mostly blocks owned by one user only) and the refcnt tree's leaf blocks merely have a pointer to this table.

\section{The filesystem module}

The filesystem module provides the standard UNIX like interface for the applications, so that applications need not be re-written. However, owing to its relaxed threat model, the file system has the following restrictions:

- Distributed filesystems like frangipani [8] and GFS [6] have a notion of a per node $\log$, which is in a universally accessible location. Any node in the cluster can replay the $\log$. In the threat model that we have chosen, the fileservers do not trust each other; so it is not possible for one fileserver to replay the log of another fileserver to restore filesystem consistency.

- Traditional filesystems have a notion of consistency in which each block in the system is in use or is free. In case of SAFIUS, this notion of consistency is tough to achieve. - Given our relaxed threat model, it is the responsibility of the fileservers to honour the filesystem structures. If they do not, there is a possibility of filesystem inconsistency. However, the SAFIUS architecture guarantees complete isolation of the effects of the misbehaving entity to its own trust domain.

\section{Read/Write control flow}

The fileservers get the root directory inode's idata during mount time. Subsequent file or directory lookups are done in the same way as in a standard UNIX filesystems.

Reads: The inode's idata fetched from the 1-hash server is the only piece of metadata that the fileserver needs to obtain from the 1-hash server. The filesystem module can fetch the blocks it wants from the storage server directly by issuing a read to the volume manager. During the read call, when the fileserver requests a shared lock on the file's inode, the 1-hash server, apart from granting the lock, also sends the idata of the inode. Using this idata it can fetch the inode block and hence the appropriate intermediate blocks and finally the leaf data block, which contains the offset requested.

Writes: Write operations from the fileserver usually proceed by first obtaining an exclusive lock on the inode. While granting the exclusive lock, the 1-hash server also sends the latest hash of the inode as a part of idata. After the update of the necessary blocks including the inode

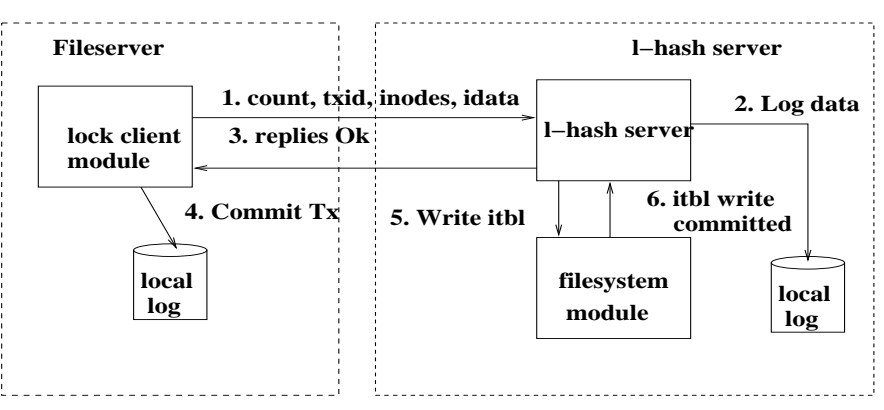

Fig. 7. Store inode data protocol

block, the hash of the new inode block corresponds to the new version of the file. As long as the idata in the 1-hash server is not updated with this, the file is still in the old version. When the new idata corresponding to this file hence inode, is updated in the i-tbl, the file moves to a new version. The 1-hash makes sure that the current user has enough permissions to update the inode's hash. Now the old data blocks and metablocks that have been replaced by new ones in the new version of the file have to be freed. The write is not visible to other fileservers until the inode's idata is updated in the 1-hash server. Since this is done before releasing the exclusive lock, any intermediate reads to the file would have to wait.

\section{N. Logging}

Journaling is used by filesystems to speed up the task of restoring the consistency of the filesystem after a crash. Many filesystems use a redo log for logging their metadata changes. During recovery after a crash, the log data is replayed to restore consistency. In SAFIUS, pending updates to the filesystem during the time of crash do not affect the consistency of the filesystem as long as the fileservers do not free any block belonging to previous version of the file and the inode's idata is not updated. When the inode's idata is updated in the l-hash server, the file moves to the next version and all subsequent accesses will see the new version of the file. The overwritten blocks have to be freed when the idata update in 1-hash server is successful and the new blocks that were written to should be freed if the idata update failed for some reason. SAFIUS uses an undo-only operation log to achieve this.

\section{O. Store inode data protocol}

To ensure that the system is consistent, the idata of an inode in the i-tbl of the 1-hash server has to be updated atomically, i.e. the inode's idata has to be either in the old state or in the new state and the fileserver that is performing the update should be able to know whether the update sent to the 1-hash server has succeeded or not. Fileservers take an exclusive lock on the file when it is opened for writ- 
ing. After flushing the modified blocks of the file and before dropping the lock it holds, the fileserver executes the store-inode data protocol with the 1-hash server to ensure consistency. The store inode data protocol is begun by the fileserver sending the count and the list of inodes and their idata to the 1-hash server. The 1-hash server stores inodes' new idata in the i-tbl atomically (either all of these inodes' hashes are updated or none of them are updated), employing a local log. It also remembers the last txid received as a part of store inode data protocol from each fileserver.

After receiving a reply from the 1-hash server, the fileserver writes a commit record to the log and commits the transaction, after which the blocks that are to be freed are queued for freeing and log space is reclaimed. The 1-hash server remembers the latest txid from the fileservers to help the fileservers know if their last execution of store inode data had succeeded. If the fileserver had crashed immediately after sending the inode's hash, it has no way of knowing whether the l-hash server received the data and had updated the i-tbl. If it had updated the i-tbl, the transaction has to be committed and the blocks meant for freeing need to be freed. If it is not the case, then the transaction has to be aborted and the blocks written as a part of that transaction have to be freed.

On recovery, the fileserver contacts the 1-hash server to get the last txid that had updated the i-tbl. If that txid does not have a commit record in the log, then the commit record is added now and the recovery procedure is started. Since all the calls to store inode data protocol are serialized within a fileserver and the global changes are visible only on updates to the i-tbl, this protocol will ensure consistency of the filesystem. The store inode data protocol takes a list of 〈inode number, idata〉 pair instead of a single inode number, idata pair. This is to ensure that dependent inodes are flushed atomically. For instance, this is useful during file creation and deletion, when the file inode is dependent on the directory inode.

\section{P. File Creation}

File creation involves obtaining a free inode number, creating a new disk inode and updating the directory entry of the parent directory with the new name-to-inode mapping. Inode numbers are generated on the fileservers autonomously without consulting any external entity. Each fileserver stores a persistent bitmap of free local inode numbers locally. This map is updated after an inode number is allocated for a new file or directory.

\section{Q. File Deletion}

Traditional UNIX systems provide a delete on close scheme for unlinks. To provide similar semantics in a dis-

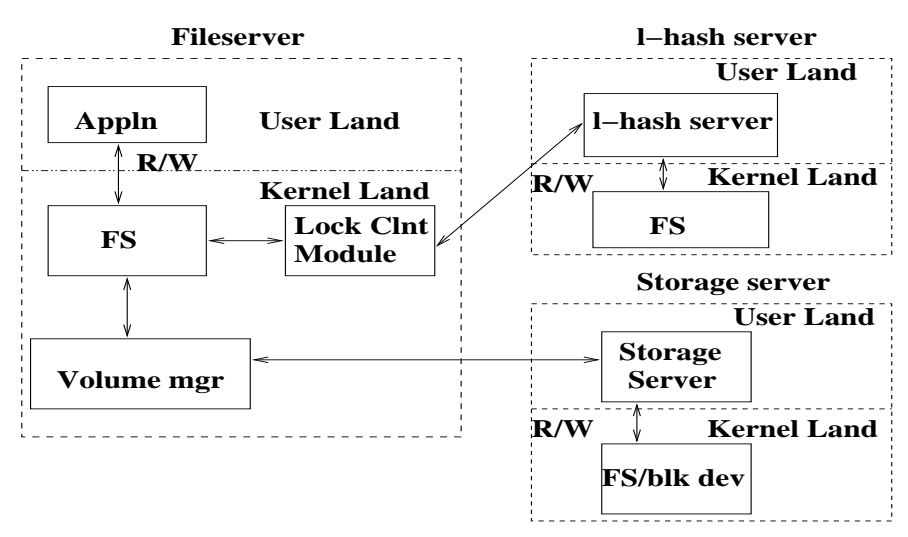

Fig. 8. Implementation Overview

tributed filesystem, one has to keep track of open references to a file from all the nodes and the file is deleted by the last process which closes the file, among all the nodes. This warrants that we need to maintain some global information regarding the open references to files. In a NFS like environment, where the server is stateless, reading and writing to a file that is unlinked from some other node results in a stale file handle error. SAFIUS' threat model does not permit similar unlink semantics. So we define a simplified unlink semantics for file deletes in SAFIUS. Unlink in SAFIUS removes the directory entry and decrements the inode reference count, but it defers deletion of the file as long as any process in the same node, from which unlink was called, has an open reference to the file. The last process on the node, from which unlink was called, deletes the file. Subsequent reads and writes from other nodes to the file do not succeed and return stale file handle error. There would not be any new reads and writes to the file from the node that called unlink as the directory entry is removed and the last process that had an open reference has closed the file. This semantics honours the standard read after write consistency. As long as the file is not deleted, a read call following a write returns the latest contents of the file. After a file is deleted, subsequent reads and writes to the file do not succeed, and hence read after write consistency.

The inode numbers have to be freed for re-allocation. As mentioned earlier, inode numbers identify the user and the machine id who owns the file. If the node which unlinks the file is same as the one which has created it, then the inode number can be marked as free in the allocation bitmap. But if unlink happens in another machine, then the fact that the inode number is free has to be communicated to that machine. Since our threat model does not assume two fileservers to trust each other, the information has to be routed through the 1-hash server. The 1-hash server sends the freed inode numbers list to the appropriate fileserver, 
during mount time (when the fileserver fetches the root directory inode's idata).

\section{R. Locking in SAFIUS}

SAFIUS uses the Memexp protocol of OpenGFS [6] with some minor modifications. The l-hash server ensures that the current uid has enough permissions to acquire the lock in the particular mode requested. The lock numbers and the inode numbers have a one to one correspondence and hence we can derive the inode number from the lock number. Using the lock number, the 1-hash server obtains the filegroup id and hence the permissions.

OpenGFS has a mechanism of callbacks wherein a node that needs a lock, currently held by another node, sends a message to that node's callback port. The node which holds the lock downgrades the lock if the lock is not in use. In SAFIUS, since the callback cannot be directly sent (the two fileservers would be mutually distrusting), the callbacks are routed through the 1-hash server.

\section{S. Lock client module}

The lock module in the fileserver handles all the client side activities of the lock protocol that was briefly described in the previous section. Apart from this, it also does the job of fetching the idata corresponding to an inode number from the 1-hash server. It also executes the store inode data protocol with the 1-hash server to ensure atomic updates of list of inodes and their idata. Figure 7 illustrates the protocol. The protocol guarantees atomicity of updates to a set of inodes and their idata.

\section{IMPLEMENTATION}

SAFIUS is implemented in the GNU/Linux environment. Figure 8 depicts the various modules in SAFIUS and their interaction. The base code used for the filesystem and lock server is OpenGFS- $0.2^{9}$ and base code used for the volume manager is GNBD-0.0.91. The Memexp lock server in OpenGFS was modified to be the l-hash server to manage locks and to store and distribute idata of the inodes. The volume manager, the filesystem module and the lock client module reside inside the kernel space, while the storage server and 1-hash server are implemented as user space processes. The current implementation of SAFIUS does not have any key management scheme and keys are manually distributed. The itbl has to reside in the untrusted storage as it has to hold the idata for all the inodes in the system. Consequently, the itbl's integrity and freshness has to be guaranteed. We achieve this by storing the itbl information in a special file in the root directory of the

\footnotetext{
${ }^{9} \mathrm{http}: / /$ opengfs.sourceforge.net/
}

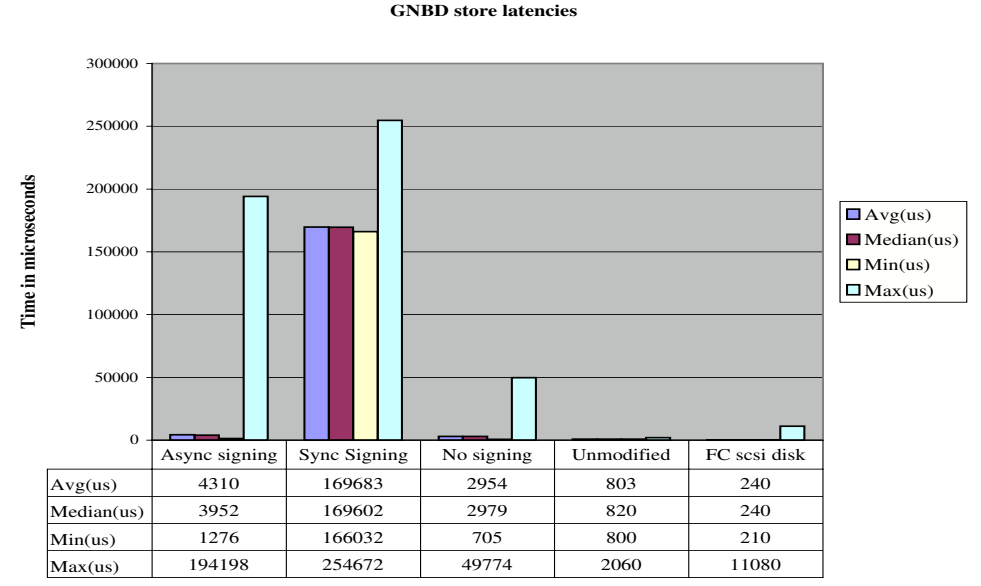

Fig. 9. Store latencies

filesystem (.itbl). The l-hash server stores the idata of this file in its local stable storage. The idata of the itbl serve as the bootstrap point for validating any file in this filesystem.

\section{Evaluation}

The performance of SAFIUS has been evaluated with the following hardware setup. The fileserver is a Pentium III $1266 \mathrm{MHz}$ machine with 896MB of physical memory. A machine with a similar configuration serves as the 1hash server, when the l-hash server and fileserver are different. A Pentium IV $1.8 \mathrm{GHz}$ machine with $896 \mathrm{MB}$ of physical memory functions as storage server. The storage server is on a Gigabit Ethernet and the fileserver and 1-hash servers are on 100Mbps Ethernet. The fileserver and 1-hash servers have a log space of 700MB on a fibre channel SCSI disk. The storage server uses a file on the ext3 filesystem (over a partition on IDE hard disk) as its store. The fileserver, storage server and the 1-hash server all run Linux kernel. The performance numbers of SAFIUS are reported in comparison with an OpenGFS setup. A GNBD device served as the shared block device. For the OpenGFS experiments, the storage server machine hosts the GNBD server and the fileserver machine hosts the OpenGFS FS client. The 1-hash server machine runs the memexp lock server of OpenGFS.

Basically two sets of configuration are studied: one in which the 1-hash server and the fileserver are the same machine and another in which the 1-hash server and the fileserver are two different machines. In OpenGFS setup the two configurations are: one in which the lock server and the FS client were in the same machine and another in which they were in two physically different machines. A thread, gfs_glockd in SAFIUS, wakes up periodically to drop the unused locks. The interval in which this thread is 


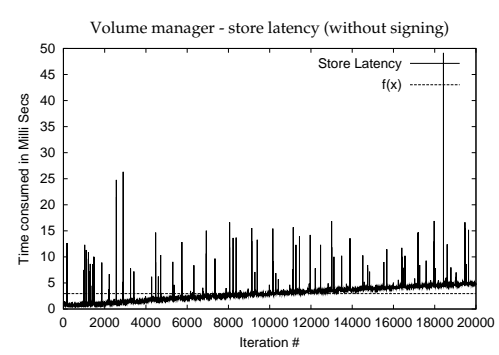

Fig. 10. No-signing

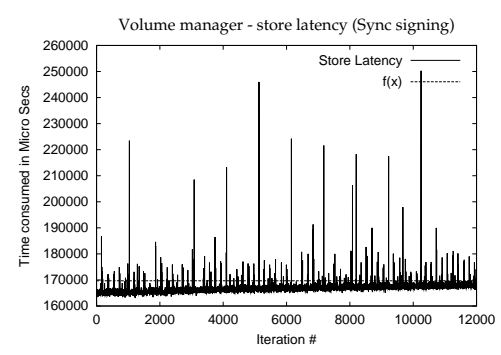

Fig. 11. Sync-signing

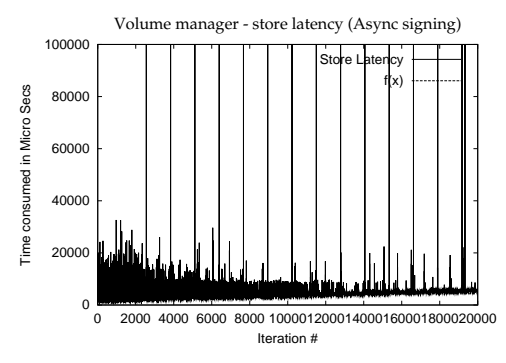

Fig. 12. Async-signing kicked in is used as a parameter of study. The performance of SAFIUS configurations are reported with and without encryption/ decryption. Hence, the performance numbers reported are for eight different combinations for SAFIUS and two different combinations for OpenGFS.

1. SAFIUS-I30: It is a SAFIUS setup in which the 1hash server and the fileserver are the same machine. The gfs_glockd interval is 30 seconds.

2. SAFIUS-I10: It is a SAFIUS setup in which the 1hash server and the fileserver are the same machine. The gfs_glockd interval is 10 seconds.

3. SAFIUS-D30: It is a SAFIUS setup in which the 1hash server and the fileserver are different machines. The gfs_glockd interval is 30 seconds

4. SAFIUS-D10: It is a SAFIUS setup in which the 1hash server and the fileserver are different machines. The gfs_glockd interval is 10 seconds

5. OpenGFS-I: It is an OpenGFS setup in which the memexp lock server and the filesystem run on the same machine

6. OpenGFS-D: It is an OpenGFS setup in which the memexp lock server and the filesystem run on different machines

SAFIUS-I30E, SAFIUS-I10E, SAFIUS-D30E, SAFIUSD10E are the SAFIUS configurations with encryption/decryption.

\section{A. Performance of Volume manager - Microbenchmark}

As described in Section II, SAFIUS volume manager uses asynchronous signing to avoid signing and verification in the common store and free path. Experiments have been conducted to measure the latencies of load and store operations. An ioctl interface in the volume manager code is used for performing loads and stores from the userland, bypassing the buffer cache of the kernel. A sequence of 20000 store operations are performed with the data from /dev/urandom. The fileserver machine is used for issuing the stores. Figure 12 shows the plot of the latency in Y-axis and the store operation sequence number along $\mathrm{X}$-axis. Approximately once every 1000 operations, there is a huge vertical line, signifying a latency of more than
$100 \mathrm{~ms}^{10}$. This is when the signer thread kicks in to perform the signing. As observed in section II, the latency of a signing operation is about $80 \mathrm{~ms}$ on a Pentium IV machine. As can be observed from figure 3 , the cost of signing is constant till the block size is $32 \mathrm{~KB}$ and grows linearly after that. This increase corresponds to the cost of SHA1 , which is amortized by the RSA exponentiation cost for smaller block sizes. The plot in figure 12 also shows the mean and median of the latencies measured. We also have studied GNBD store latencies for various combinations. The results are reported in Fig. 9. As to be expected, synchronous signing incurs the highest overhead while the asynchronous signing, on an average, appears to be only $30 \%$ costlier compared to no-signing.

The next subsection describes the performance studies conducted for the filesystem module.

\section{B. Filesystem Performance - Microbenchmark and Popu- lar benchmarks}

The performance of the filesystem module has been analyzed by running the Postmark ${ }^{11}$ microbenchmark suite and compilation of OpenSSH, OpenGFS and Apache web server sources. As described in the beginning of this section, numbers are reported for six configurations. Figure 16 shows the numbers obtained by running Postmark suite. The Postmark benchmark has been run with the following configuration:

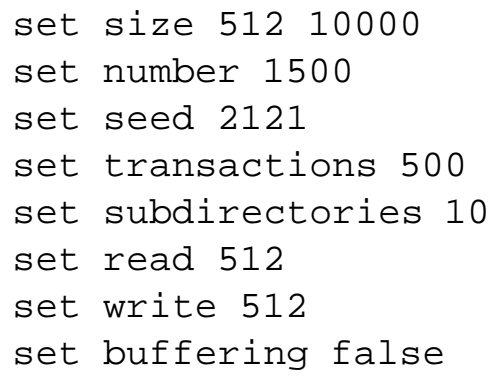

Currently, owing to the simple implementation of the storage server, logical to physical lookups take a lot of time

\footnotetext{
${ }^{10}$ The Y-axis scale is trimmed, the latencies are about $170 \mathrm{~ms}$

${ }^{11}$ Benchmark from Network Appliance
} 


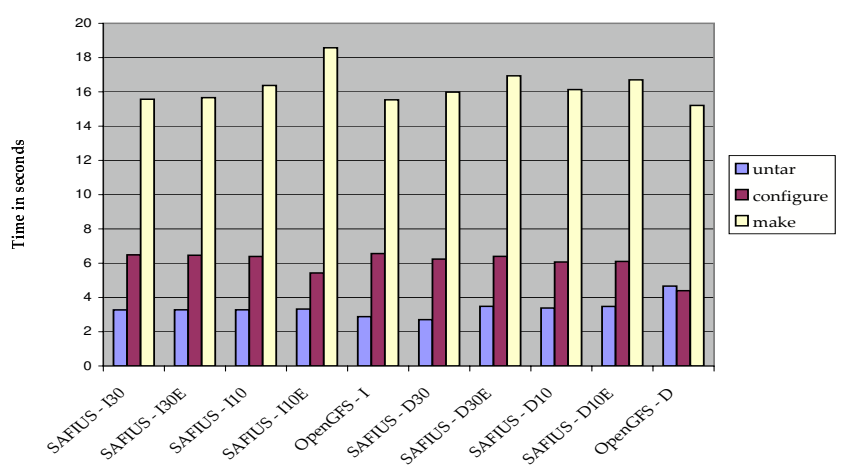

Fig. 13. Apache compilation

and hence Postmark with bigger configurations take a long time to complete. Hence we report Postmark results only for the smaller configurations. SAFIUS beats OpenGFS in metadata intensive operations like create and delete. Creations and deletions in SAFIUS are not immediately committed (even to $\log$ ) and are committed only when the lock is dropped and hence the explanation. With the current input configuration, reads and appends for SAFIUS-D30, SAFIUS-I30, OpenGFS-I and OpenGFS-D seem to be the same. Bigger configurations may show some difference. SAFIUS-I10 and OpenGFS-D10 seem to perform poorly for appends and reads due to flushing of data belonging to files that would anyway get deleted. There is no difference between the 1-hash server being in the same machine or in different machine for Postmark suite. For the Postmark suite, there is not much difference between the configurations that has encryption/decryption and the ones that doesn't have.

Next three performance tests involved compilation of OpenSSH, OpenGFS and Apache web server. Three activities were performed on the source tree: untar (tar zxvf) of source, configure and make. Time taken for each of these operations for all the ten configurations described before is reported. Figure 14 is the result of OpenSSH compilation. All SAFIUS configurations take twice the amount of time for untar compared to OpenGFS-I, while OpenGFSD takes four times the time taken by OpenGFS-I for untar. OpenSSH compilation did not complete in SAFIUSI10E. Time taken for make in SAFIUS-D30 and SAFIUSD30E, seems to be almost same, while SAFIUS-I30E takes $10 \%$ more time than SAFIUS-I30. The best SAFIUS configurations (SAFIUS-D30 and SAFIUS-D30E) are within $120 \%$ of the best OpenGFS configuration (OpenGFSD). The worst SAFIUS configurations (SAFIUS-I10E and SAFIUS-D10E) are within $200 \%$ of worst OpenGFS configuration (OpenGFS-I). The next performance test was
OpenSSH compilation

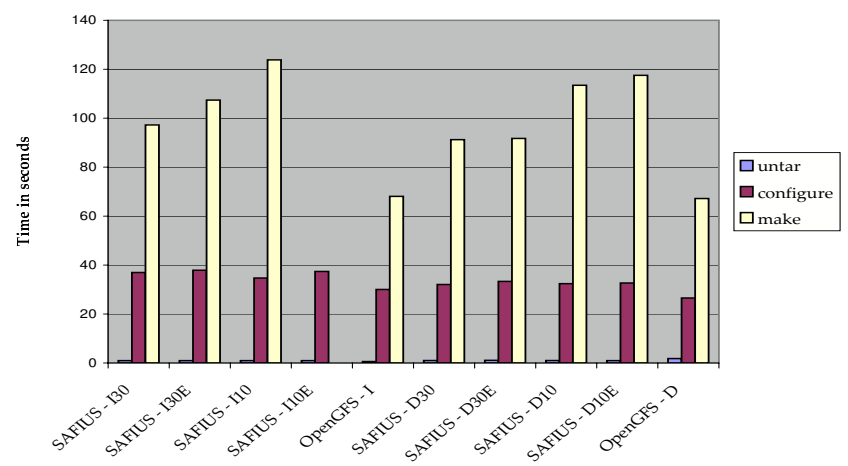

Fig. 14. OpenSSH compilation

compilation of Apache source. Figure 13 shows the time taken for untar, configure and make operations of Apache source compilation. SAFIUS-D30 gives the best performance for untar and OpenGFS-D gives the worst. This is probably because of reduced interference for syncing the idata writes. OpenGFS-D does the best for make and configure. Among SAFIUS configurations, SAFIUS-D10 does the best for configure and SAFIUS-I30 does the best for make. The last performance test is OpenGFS compilation. make was run from the $\mathrm{src} / \mathrm{fs}$ subtree instead of the toplevel source tree. Figure 15 shows the time taken for untar, configure and make operations for compiling OpenGFS. SAFIUS-I30E and SAFIUS-I10E takes about $120 \%$ of time taken by SAFIUS-I30 and SAFIUSI10 respectively for running configure and make. The best SAFIUS configuration for running make (SAFIUS-D30) takes around $115 \%$ of time taken by best OpenGFS configuration (OpenGFS-I). The worst SAFIUS configuration for running make (SAFIUS-I30E) takes about $115 \%$ of time taken by worst OpenGFS configuration (OpenGFSD).

We can conclude, from the experiments run, that SAFIUS seems to be comparable (or sometimes better) to OpenGFS for metadata intensive operations and around $125 \%$ of the best OpenGFS configuration without encryption/decryption, and around $150 \%$ of the best OpenGFS configuration with encryption/decryption and for other operations.

\section{CONCLUSiOns \& Future Work}

In this work the design and implementation of a secure distributed filesystem over untrusted storage was discussed. SAFIUS provides confidentiality, integrity, freshness and accountability guarantees, protecting the clients from malicious storage and the storage from malicious clients. SAFIUS requires that trust be placed on the lock- 

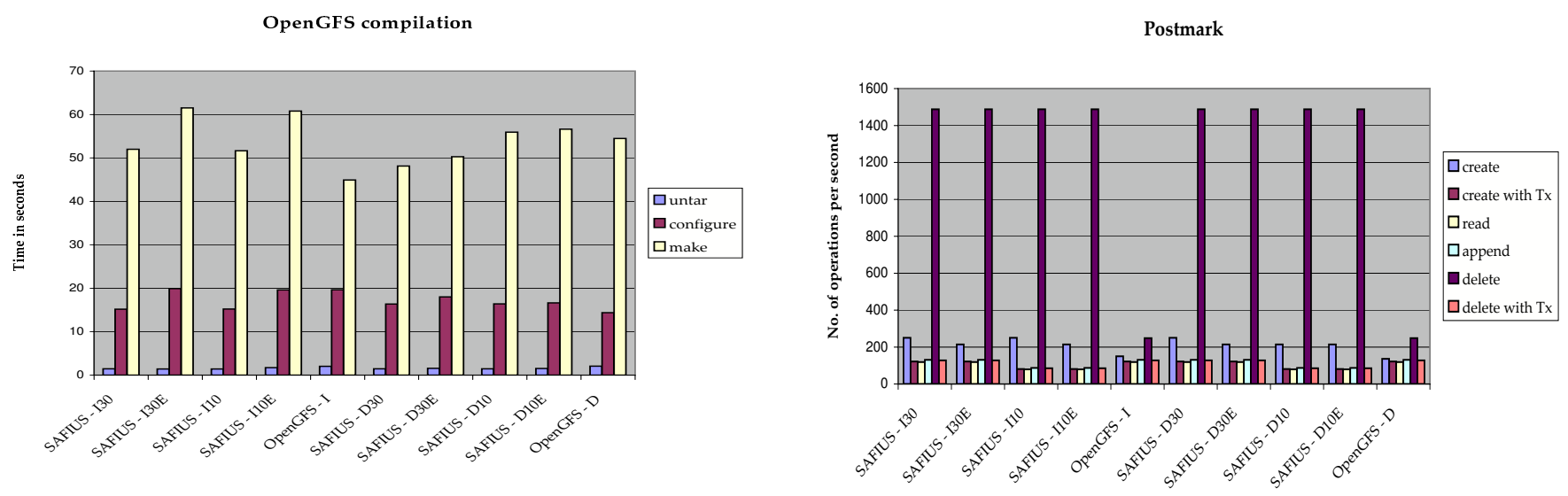

Fig. 15. OpenGFS compilation

server (l-hash server), to provide all the security guarantees; a not so unrealistic threat model. For the applications, SAFIUS is like any other filesystem; it does not require any change of interfaces and hence has no compatibility issues. SAFIUS uses the $l$-hash server to store and retrieve the hash codes of the inode blocks. The hash codes reside on the untrusted storage and the integrity of the system is provided with the help of a secure local storage in the $l$ hash server. SAFIUS is flexible; users choose which client fileservers to trust and how long. SAFIUS provides ease of administration; the fileservers can fail and recover without affecting the consistency of the filesystem and without the involvement of another entity. With some minor modifications, SAFIUS can easily provide consistent snapshots of the filesystem (by not deleting the overwritten blocks). The performance of SAFIUS is promising given the security guarantees it provides. A detailed performance study (under heavier loads), has to be done in-order to establish the consistency in performance.

Possible avenues for future work are:

- Fault tolerant distributed 1-hash server: The 1-hash server in SAFIUS can become a bottleneck and prevent scalability of fileservers. It would be interesting to see how the system performs when we have a fault tolerant distributed 1-hash server in place of existing 1-hash server. The distributed lock protocol should work without assuming any trust between fileservers.

- Optimizations in storage server: The current implementation of the storage server is a simple request response protocol that serializes all the requests. It would be a performance boost to do multiple operations in parallel. This may affect the write ordering assumptions that exist in the current system.

- Utilities: Userland filesystem debug utilities and failure recovery utilities have to be written.

- Key management: SAFIUS does not have a key man-
Fig. 16. Postmark

agement scheme and no interfaces by which the users can communicate to the fileservers their keys. This would be an essential element for the system.

The source code for SAFIUS is available on request.

\section{REFERENCES}

[1] Kevin Fu, M. Frans Kaashoek, and David Mazieres. Fast and secure distributed read-only file system. Computer Systems, 20(1):124, 2002.

[2] D. Hitz, J. Lau, and M. Malcolm. File system design for an NFS file server appliance. In Proceedings of the USENIX Winter 1994 Technical Conference, pages 235-246, San Fransisco, CA, USA, 1994.

[3] David Mazires Jinyuan Li, Maxwell Krohn and Dennis Shasha. Secure untrusted data repository (sundr). Technical report, NYU Department of Computer Science, 2003.

[4] M. Kallahalla, E. Riedel, R. Swaminathan, Q. Wang, and K. Fu. Plutus - scalable secure file sharing on untrusted storage. In In Proceedings of the Second USENIX Conference on File and Storage Technologies (FAST). USENIX, March 2003.

[5] Athicha Muthitacharoen, Benjie Chen, and David Mazieres. A low-bandwidth network file system. In Symposium on Operating Systems Principles, pages 174-187, 2001.

[6] Steven R. Soltis, Thomas M. Ruwart, and Matthew T. O'Keefe. The Global File System. In Proceedings of the Fifth NASA Goddard Conference on Mass Storage Systems, pages 319-342, 1996.

[7] Christopher A. Stein, John H. Howard, and Margo I. Seltzer. Unifying file system protection. In In Proc. of the USENIX Technical Conference, pages 79-90, 2001.

[8] Chandramohan A. Thekkath, Timothy Mann, and Edward K. Lee. Frangipani: A scalable distributed file system. In Symposium on Operating Systems Principles, pages 224-237, 1997.

[9] R. Vingralek U. Maheshwari and B. Shapiro. How to build a trusted database system on untrusted storage. In OSDI: 4th Symposium on Operating Systems Design and Implementation, 2002. 